\title{
High-Order Methods for Systems of Fractional Ordinary Differential Equations and Their Application to Time-Fractional Diffusion Equations
}

\author{
Luís L. Ferrás • Neville Ford • \\ Maria Luísa Morgado • Magda Rebelo
}

Received: 17 July 2019 / Accepted: 13 September 2019

(C) Springer Nature Switzerland AG 2020

\begin{abstract}
Taking into account the regularity properties of the solutions of fractional differential equations, we develop a numerical method which is able to deal, with the same accuracy, with both smooth and nonsmooth solutions of systems of fractional ordinary differential equations of the Caputo-type. We provide the error analysis of the numerical method and we illustrate its feasibility and accuracy through some numerical examples. Finally, we solve the time-fractional diffusion equation using a combination of the method of lines and the newly developed hybrid method.
\end{abstract}

Keywords Fractional diffusion - Caputo derivative $\cdot$ Nonpolynomial collocation method P Polynomial collocation method $\cdot$ Method of lines

Mathematics Subject Classification $45 \mathrm{~K} 05 \cdot 65 \mathrm{~L} 20 \cdot 65 \mathrm{M} 12 \cdot 65 \mathrm{R} 20$

\section{Introduction}

Nowadays, fractional differential equations are recognized as fundamental tools in the modeling of many phenomena in science and engineering $[29,30,33,36]$.

\footnotetext{
L. L. Ferrás

Department of Mathematics, Centre of Mathematics (CMAT), University of Minho, Campus de Azurém, 4800-058 Guimarães, Portugal

e-mail: luislimafr@gmail.com

N. Ford

Department of Mathematics, University of Chester, Chester CH1 4BJ, UK

e-mail: njford@ chester.ac.uk

M. L. Morgado $(\varangle)$

Center for Computational and Stochastic Mathematics, Instituto Superior Técnico, Universidade de Trás-os-Montes e Alto Douro, Departamento de Matemática, UTAD Quinta de Prados, 5001-801 Vila Real, Portugal

e-mail: luisam@utad.pt

\section{Rebelo}

Departamento de Matemática, Faculdade de Ciências e Tecnologia and Centro de Matemática e Aplicações (CMA), Universidade NOVA de Lisboa, Quinta da Torre, 2829-516 Caparica, Portugal

e-mail: msjr@fct.unl.pt
} 
Usually, analytical solutions for fractional differential equations are not available and, in the cases where these can be obtained, they are given in terms of series representations, which makes them difficult to handle. Therefore, numerical methods are crucial for this kind of problems.

Here, we are initially concerned with the numerical solution of systems of fractional ordinary differential equations of the Caputo type:

$$
\begin{aligned}
D^{\alpha} \mathbf{y}(t) & =A \mathbf{y}(t)+\mathbf{F}(t), \quad t \in(0, T] \\
\mathbf{y}(0) & =\mathbf{y}_{\mathbf{0}},
\end{aligned}
$$

where $A$ is a constant matrix $A=\left[a_{i j}\right]_{i, j=1, \ldots, n}, \mathbf{y}(t)=\left[y_{1}(t) y_{2}(t) \ldots y_{n}(t)\right]^{T}, \mathbf{F}(t)=\left[f_{1}(t) f_{2}(t) \ldots f_{n}(t)\right]^{T}$ and $\mathbf{y}_{\mathbf{0}}=\left[\begin{array}{llll}y_{01} & y_{02} \ldots y_{0 n}\end{array}\right]^{T}$, where $y_{0 i}=y_{i}(0), i=1, \ldots, n$.

The theory and numerical analysis of this type of problems is well described in the literature [5,7-11], however, we believe that due to the features of fractional calculus, there is still a lack of highly accurate and efficient numerical methods for this kind of differential equations. First, because fractional differential operators are non-local and, as a consequence, in order to compute the solution at a certain time, all the previous time levels must have been computed and stored previously, a fact that results in a high computational effort. Second, because fractional differential equations usually exhibit singularities at the origin in time, and therefore, the order of convergence of the numerical schemes may decrease significantly whenever the unknown solution does not satisfy the demanded regularity assumptions (see for Example [34] and the survey paper [13]).

Here, we will mainly focus on this second aspect, and we will present a numerical method which is able to attain the same accuracy with both smooth and nonsmooth solutions. This follows the investigation initiated in [14]. In that paper, the convergence analysis has not been provided, although the numerical examples confirm the expected orders of convergence. Here, we will consider a modification of this algorithm. Because the potential singularities of the solution arise at the origin, instead of using nonpolynomial approximation in all of the time domain, we consider an 'hybrid' collocation method, which consists of a nonpolynomial approximation near the origin, reflecting the singular behavior of the solution near that point, and in the remaining domain, we use a polynomial approximation. As it is natural and shown later, this reduces the computational effort.

The numerical technique used to solve these systems of fractional ordinary differential equations, can also be used to solve the time-fractional diffusion equation, that have shown to be useful in the modeling of anomalous diffusion processes, in which the classical (integer order) diffusion equations can not provide an accurate description of such anomalous behaviors [32]. Time-fractional diffusion equations, with the order of the time derivative between 0 and 1 , correspond to sub-diffusive models, in which, the mean square displacement of the diffusive particle is proportional to $t^{\alpha}$, being $\alpha$ the order of the time derivative. In the case where $\alpha=1$, we recover the classical diffusion equation, the case where it is assumed that the mean square displacement of a particle is proportional to the time $t[16,26-28]$.

Therefore, in this work we are also concerned with the numerical solution of the one-dimensional time-fractional diffusion equation:

$\frac{\partial^{\alpha} u(x, t)}{\partial t^{\alpha}}=D_{\alpha} \frac{\partial^{2} u(x, t)}{\partial x^{2}}+f(x, t), \quad t \in(0, T], \quad 0<x<L$,

with initial condition:

$u(x, 0)=g(x)$,

and boundary conditions:

$u(0, t)=u_{0}, \quad u(L, t)=u_{L}$,

where we assume that $D_{\alpha}, u_{0}$ and $u_{L}$ are constants, the order of the fractional derivative satisfies $0<\alpha<1$, and the fractional derivative is again given in the Caputo sense [6]. Note that $D_{\alpha}$ stands for a general diffusion coefficient with dimensions $[\text { length }]^{2} /[\text { time }]^{\alpha}$.

Finite difference methods are the most commonly found for the numerical solution of this kind of problems (see for example [2-4,15,19-21,25,38-40]) although other numerical approaches have also appeared, such as, for 
example, finite element methods [35,37], meshless collocation methods [17] and collocation spectral methods [18]. Again, these methods are computationally demanding and they do not take into account the potential singularities of the solution. Therefore, here we will use the method of lines (MOL) in combination with the 'hybrid' method for systems of ordinary differential equations.

The paper is organized in the following way: in Sect. 2 we describe the numerical method for systems of fractional ordinary differential equations and we present the error analysis. In Sect. 3 we present some numerical results, and study the numerical solution of the time-fractional diffusion equation. We end with the main conclusions.

For details on the existence and uniqueness of solution for this type of equation please see [5,6,12,22-24].

\section{A Hybrid Nonpolynomial Collocation Method for Fractional Ordinary Differential Systems}

Let us first recall a well known result (see [6]) about the regularity properties of the solution of single order ordinary differential equations of the form:

$$
\begin{aligned}
D^{\alpha} y(t) & =f(t, y(t)), \quad t \in(0, T], \\
y(0) & =y_{0},
\end{aligned}
$$

with $\alpha>0$.

Theorem 1 (Existence) Let $K, g, \alpha>0$ and $y_{0} \in \mathbb{R}$. Define $G:=[0, g] \times\left[y_{0}-K, y_{0}+K\right]$ and let the function $f: G \rightarrow \mathbb{R}$ be continuous. Furthermore, define $M:=\sup _{(x, z) \in G}|f(x, z)|$ and

$g *=\left\{\begin{array}{cc}g & \text { if } M=0 \\ \min \left\{g,(K \Gamma(\alpha+1) / M)^{1 / \alpha}\right\} & \text { else. }\end{array}\right.$

Then, there exists a function $y \in C[0, g *]$ solving the initial value problem (6)-(7).

Theorem 2 (Uniqueness) Let $K, g, \alpha>0$ and $y_{0} \in \mathbb{R}$. Define $G:=[0, g] \times\left[y_{0}-K, y_{0}+K\right]$ and let the function $f: G \rightarrow \mathbb{R}$ be continuous and fulfil a Lipschitz condition with respect to the second variable, that is, $\left|f\left(x, y_{1}\right)-f\left(x, y_{2}\right)\right| \leq L\left|y_{1}-y_{2}\right|$, with some constant $L>0$ independent of $x, y_{1}$ and $y_{2}$. Then, denoting $g *$ as in previous Theorem (1), there exists a uniquely defined function $y \in C[0, g *]$ solving the initial value problem (6)-(7).

Lemma 1 Assume that the solution y of (6)-(7) exists and is unique on $[0, T]$, for a certain $T>0$. Let $\alpha=\frac{p}{q}$, where $p \geq 1$ and $q \geq 2$ are two relatively prime integers and let the right-hand side function $f$ to be written in the form $f(t, y(t))=\bar{f}\left(t^{1 / q}, y(t)\right)$, where $\bar{f}$ is analytic in a neighborhood of $(0, y(0))$, then the unique solution of the problem (6)-(7) can be represented in terms of powers of $t^{1 / q}$ :

$y(t)=\sum_{k=0}^{\infty} a_{k} t^{k / q}, t \in[0, r)$,

where $r<T$ and $a_{k}, k \geq 0$, are constants.

Remark 1 1. First, it should be noted that we assume $\alpha$ to be rational. If not, we can chose a close rational approximation and solve for that.

2. Second, from the above Lemma it follows that for $m \in \mathbb{N}$ fixed, the solution of (6)-(7) can be written in the form $y(t)=y^{(1)}(t)+y^{(2)}(t)$, where $y^{(1)} \in C^{m}([0, T])$, for a certain $m \geq 1$, and $y^{(2)}$ is the nonsmooth part of $y$.

Obviously, this result also holds if instead of a single differential equation, we have a system of multiple equations. 
Also, remember that assuming that the right hand-side function $f(t, y)$ is continuous on $[0, T] \times \mathbb{R}$, then the differential equation (6)-(7) is equivalent to the singular Volterra integral equation [6]:

$y(t)=y_{0}+\frac{1}{\Gamma(\alpha)} \int_{0}^{t}(t-s)^{\alpha-1} f(s, y(s)) d s, \quad t \in(0, T]$.

Based on the previous equivalence and Lemma 1, we present a numerical method for linear systems of ordinary differential equations (1)-(2).

In order to approximate the solution of (1)-(2) we consider a nonuniform mesh on [0,T], as in [1]. Given $N \in \mathbb{N}$, let $i_{0}$ be an integer such that $\left(\frac{N}{i_{0}}\right)^{\frac{m}{\alpha}} \leq N$ and $\left(\frac{N}{i_{0}-1}\right)^{\frac{m}{\alpha}}>N$ and let $N^{\prime}=N-i_{0}+1$. The partition on $[0, T]$ is defined through the meshpoints:

$t_{0}=0, t_{i}=\left(\frac{i_{0}+i-1}{N}\right)^{\frac{m}{\alpha}} T, \quad i=1,2, \ldots, N^{\prime}-1$,

and the $N^{\prime}$ subintervals are given by:

$\sigma_{0}=\left[0, t_{1}\right], \quad \sigma_{i}=\left(t_{i}, t_{i+1}\right], i=1,2, \ldots, N^{\prime}-1$,

with lengths $\tau_{i}=t_{i+1}-t_{i}, i=0,1, \ldots, N^{\prime}-1$. Define also $\tau=\max \left\{\tau_{i}, i=0,1, \ldots, N^{\prime}-1\right\}$.

Note that the integer $i_{0}$ satisfies the condition

$N^{1-\alpha / m} \leq i_{0} \leq N(N-1)^{-\alpha / m}$,

and from this inequality we can prove that there exists a positive constant $c$ such that [1]

$\tau_{i} \leq c\left(i+i_{0}-2\right)^{\frac{m-\alpha}{\alpha}} N^{-\frac{m}{\alpha}} \leq c N^{-1}, i=0,1, \ldots, N^{\prime}-1$.

Consider the space

$\mathcal{V}_{m}^{\alpha}=\operatorname{span}\left\{t^{i+j \alpha}, i, j \in \mathbb{N}_{0}, i+j \alpha<m\right\}=\operatorname{span}\left\{t^{\nu_{k}}, k=1, \ldots, \ell\right\}, \quad \ell=\# \mathcal{V}_{m}^{\alpha}$.

Taking Lemma 1 into account, if near the origin we approximate the solution of (1)-(2) with a function spanned by elements of space $\mathcal{V}_{m}^{\alpha}$, then it will reflect the potential nonsmooth properties of the solution near the singularity. Therefore, for each $m \in \mathbb{N}$, we define the space

$S_{\tau}^{m}([0, T])=\left\{u \in C([0, T]):\left.\left.u\right|_{\sigma_{0}} \in \mathcal{V}_{m}^{\alpha}\right|_{\sigma_{0}},\left.\left.u\right|_{\sigma_{l}} \in \mathcal{P}_{m-1}\right|_{\sigma_{l}}, l=1,2, \ldots, N^{\prime}-1\right\}$,

where $\mathcal{P}_{m-1}$ is the space of polynomials of degree less than or equal to $m-1$ and $\sigma_{l}, l=0,1, \ldots ., N^{\prime}-1$, are defined by (12).

The idea of the method is to approximate the solution of (1)-(2) by a function $v$ such that $v \in S_{\tau}^{m}([0, T])$. In order to define $v$ we proceed as follows.

On the first interval of the partition, $\sigma_{0}$, we define $\ell$ collocation points $t_{0 j}=c_{j} \tau_{0}, j=1, \ldots, \ell, c_{j} \in[0,1]$, and on the remaining intervals $\sigma_{l}, l=1, \ldots, N^{\prime}-1$, we consider $m$ collocation points $t_{l j}=t_{l}+c_{j} \tau_{l}, \quad j=$ $1, \ldots, m, c_{j} \in[0,1]$. Noting that each equation of system (1) can be written as

$y_{i}(t)=y_{0 i}+\frac{1}{\Gamma(\alpha)} \int_{0}^{t}(t-s)^{\alpha-1}\left(\sum_{k=1}^{n} a_{i k} y_{k}(s)+f_{i}(s)\right) d s$,

we will then seek for a function $\mathbf{v}(t)=\left[v_{1}(t) v_{2}(t) \ldots v_{n}(t)\right]^{T}$ such that $v_{i} \in S_{\tau}^{m}([0, T]), i=1,2, \ldots, n$, that satisfies

$$
\begin{gathered}
v_{i}\left(t_{0 j}\right)=y_{0 i}+\frac{1}{\Gamma(\alpha)} \int_{0}^{t_{0 j}}\left(t_{0 j}-s\right)^{\alpha-1}\left(\sum_{k=1}^{n} a_{i k} v_{k}(s)+f_{i}(s)\right) d s, j=1, \ldots, \ell, \\
v_{i}\left(t_{p j}\right)=y_{0}+\frac{1}{\Gamma(\alpha)} \int_{0}^{t_{p j}}\left(t_{p j}-s\right)^{\alpha-1}\left(\sum_{k=1}^{n} a_{i k} v_{k}(s)+f_{i}(s)\right) d s, \\
p=1, \ldots, N^{\prime}-1, j=1, \ldots, m .
\end{gathered}
$$


In order to obtain approximations for each $v_{i}\left(t_{0 j}\right), i=1, \ldots, n, j=1, \ldots, \ell$, we define the Lagrange functions, $\left.\left.\mathcal{L}_{0 j}\right|_{\sigma_{0}} \in \mathcal{V}_{m}^{\alpha}\right|_{\sigma_{0}}, j=1, \ldots, \ell$, such that

$\mathcal{L}_{0 j}\left(t_{0 k}\right)=\delta_{j k}, k=1, \ldots, \ell$.

Then, we can write

$\mathcal{L}_{0 j}(t)=\sum_{i=1}^{\ell} \beta_{j i} t^{\nu_{i}}$,

where, for each $j=1, \ldots, \ell$, the coefficients $\beta_{j i}$ may be obtained by solving the linear system (17).

It will be convenient to introduce the following projection operator $P_{\tau}:\left.C([0, T]) \rightarrow \mathcal{V}_{m}^{\alpha}\right|_{\sigma_{0}}$, defined by (see [1]): $\left(P_{\tau} g\right)(s)=\sum_{k=1}^{\ell} \mathcal{L}_{0 k}(s) g\left(t_{0 k}\right), \quad s \in \sigma_{0}=\left[0, \tau_{0}\right]$.

Hence, for $t \in \sigma_{0}$, we use the following representation for $\left.v_{i} \in \mathcal{V}_{m}^{\alpha}\right|_{\sigma_{0}}, i=1, \ldots, n$ :

$v_{i}(t)=\sum_{k=1}^{\ell} v_{i}\left(t_{0 k}\right) \mathcal{L}_{0 k}(t)$.

On the remaining subintervals of the partition, $\sigma_{j}, j=1, \ldots, N^{\prime}-1$, each $y_{i}$ will be approximated by $v_{i} \in \mathcal{P}_{m-1}$ :

$v_{i}(t)=\sum_{\gamma=1}^{m} L_{j \gamma}(t) v_{i}\left(t_{j \gamma}\right), \quad t \in \sigma_{j}$,

where $L_{j \gamma}, j=1, \ldots, N^{\prime}-1, \gamma=1, \ldots, m$, are the Lagrange polynomials associated with the collocations points $t_{j \gamma}=t_{j}+\tau_{j} c_{\gamma}$, defined by

$L_{j \gamma}(t)=\prod_{\substack{p=1 \\ p \neq \gamma}}^{m} \frac{t-t_{j p}}{t_{j \gamma}-t_{j p}}$.

We also define the operators $P_{j}: C([0, T]) \rightarrow \mathcal{P}_{m-1}$ by

$$
\left(P_{j} g\right)(s)=\sum_{\gamma=1}^{m} L_{j \gamma} g\left(t_{j \gamma}\right), \quad s \in \sigma_{j}, j=1, \ldots, N^{\prime}-1 .
$$

The values $v_{i}\left(t_{0 k}\right), k=1, \ldots, \ell$ and $v_{i}\left(t_{l k}\right), l=1, \ldots N^{\prime}-1, k=1, \ldots, m$, with $i=1, \ldots, n$, are obtained by imposing that the functions $v_{i}(t)$ at the collocation points satisfy the integral equations:

$$
\begin{aligned}
v_{i}\left(t_{0 j}\right)= & y_{0 i}+\frac{1}{\Gamma(\alpha)} \int_{0}^{t_{0 j}}\left(t_{0 j}-s\right)^{\alpha-1}\left(\sum_{p=1}^{n} a_{i p} \sum_{\gamma=1}^{\ell} v_{p}\left(t_{0 \gamma}\right) \mathcal{L}_{0 \gamma}(s)+f_{i}(s)\right) d s, j=1, \ldots, \ell, \\
v_{i}\left(t_{l k}\right)= & y_{0 i}+\frac{1}{\Gamma(\alpha)} \int_{0}^{t_{1}}\left(t_{l k}-s\right)^{\alpha-1}\left(\sum_{p=1}^{n} a_{i p} \sum_{\gamma=1}^{\ell} v_{p}\left(t_{0 \gamma}\right) \mathcal{L}_{0 \gamma}(s)\right) d s \\
& +\frac{1}{\Gamma(\alpha)} \sum_{j=1}^{l-1} \int_{t_{j}}^{t_{j+1}}\left(t_{l k}-s\right)^{\alpha-1}\left(\sum_{p=1}^{n} a_{i p} \sum_{\gamma=1}^{m} L_{j \gamma}(s) v_{p}\left(t_{j \gamma}\right)\right) d s \\
& +\frac{1}{\Gamma(\alpha)}\left(\int_{t_{l}}^{t_{l k}}\left(t_{l k}-s\right)^{\alpha-1}\left(\sum_{p=1}^{n} a_{i p} \sum_{\gamma=1}^{m} L_{l \gamma}^{(k)}(s) v_{p}\left(t_{l}+\tau c_{k} c_{\gamma}\right)\right) d s+\int_{0}^{t_{l k}}\left(t_{l k}-s\right)^{\alpha-1} f_{i}(s) d s\right), \\
l= & 1, \ldots, N^{\prime}-1, k=1, \ldots, m,
\end{aligned}
$$


where $L_{l \gamma}^{(k)}, l=1, \ldots, N^{\prime}-1, \gamma=1, \ldots, m$, are the Lagrange polynomials associated with the points $t_{l}+\tau_{l} c_{\gamma} c_{k}$ defined similarly to (20).

After solving (22) and (2), the approximate solution of system (1)-(2), $\mathbf{v}(t)=\left[v_{i}(t)\right]_{i=1}^{n}$ is given by:

$v_{i}(t)= \begin{cases}\sum_{k=1}^{\ell} v_{i}\left(t_{0 k}\right) \mathcal{L}_{0 k}(t), \quad t \in \sigma_{0}, \\ \sum_{k=1}^{m} v_{i}\left(t_{j k}\right) L_{j k}(t), \quad t \in \sigma_{j}, j=1, \ldots, N^{\prime}-1 .\end{cases}$

\subsection{Convergence Analysis}

In what follows, we present the convergence analysis of the hybrid method presented for ordinary fractional order systems. In order to do that, we first introduce some notation and some useful lemmas.

For each vector $x$, and matrix $A$, we denote

$\|x\|=\|x\|_{\infty}=\max _{i}\left\{\left|x_{i}\right|\right\}, \quad\|A\|=\|A\|_{\infty}=\max _{i}\left\{\sum_{j}\left|a_{i j}\right|\right\}$

and for $f \in C([a, b])$ we define the maximum norm of a continuous function defined by $\|f\|_{[a, b]}=\max _{t \in[a, b]}|f(t)|$.

In the convergence analysis we shall need an auxiliary lemma from [1].

Theorem 3 Let $\mathcal{L}_{0 k}, k=1, \ldots, \ell$, be the Lagrange functions defined by (17) and $\sigma_{0}=\left[0, t_{1}\right]=\left[0, \tau_{0}\right]$. There exists a positive constant $\Delta_{0}$ such that

$\left\|\mathcal{L}_{0 k}\right\|_{\sigma_{0}} \leq \Delta_{0}, \quad k=1, \ldots, \ell$.

Furthermore, given $m \in \mathbb{N}$ and $f(t)=f_{1}(t)+f_{2}(t)$, where $f_{1} \in C^{m}([0, T])$ and $f_{2} \in V_{m}^{\alpha}$, we have

$\left\|f-P_{\tau} f\right\|_{\sigma_{0}} \leq \bar{c} \tau_{0}^{m}\left\|f_{1}^{(m)}\right\|_{\sigma_{0}}$,

for some positive constant $\bar{c}$.

We now provide an estimate for the error at the first subinterval of the mesh. For each $j=1, \ldots, \ell$, we analyse the error at the collocation points $t_{0 j}$ :

$\mathbf{e}_{0 j}=\left[e_{0 j}^{1} e_{0 j}^{2} \ldots e_{0 j}^{n}\right]^{T}$,

where $e_{0 j}^{i}=y_{i}\left(t_{0 j}\right)-v_{i}\left(t_{0 j}\right), i=1,2, \ldots, n$.

Lemma 2 Let $\mathbf{y}(t)=\left[y_{k}(t)\right]_{k=1}^{n}$ be the solution of $(1)-(2)$, and $\mathbf{v}(t)=\left[v_{k}(t)\right]_{k=1}^{n}$ the approximate solution obtained by the hybrid collocation method and defined by (24). On the subinterval $\sigma_{0}$, we have

$\max _{1 \leq k \leq n}\left\|y_{k}-v_{k}\right\|_{\sigma_{0}} \leq C N^{-m}$,

where $C$ is a positive constant that does not depend on $N$.

Proof Taking (14) and (15) into account, we have, for $i=1, \ldots, n$ and $j=1, \ldots, l$,

$$
\begin{aligned}
\left|e_{0 j}^{i}\right|=\left|y_{i}\left(t_{0 j}\right)-v_{i}\left(t_{0, j}\right)\right| & \leq \frac{1}{\Gamma(\alpha)} \int_{0}^{t_{0 j}}\left(t_{0 j}-s\right)^{\alpha-1} \sum_{k=1}^{n}\left|a_{i k}\right|\left|y_{k}(s)-v_{k}(s)\right| d s \\
& \leq \frac{1}{\Gamma(\alpha)} \sum_{k=1}^{n}\left|a_{i k}\right|\left\|y_{k}-v_{k}\right\|_{\sigma_{0}} \int_{0}^{t_{0 j}}\left(t_{0 j}-s\right)^{\alpha-1} d s \\
& \leq \frac{t_{0 j}^{\alpha}}{\Gamma(\alpha+1)} \sum_{k=1}^{n}\left|a_{i k}\right|\left\|y_{k}-v_{k}\right\|_{\sigma_{0}}
\end{aligned}
$$


Let us now analyse $\left\|y_{k}-v_{k}\right\|_{\sigma_{0}}, k=1, \ldots, n$. First, note that

$\left\|y_{k}-v_{k}\right\|_{\sigma_{0}} \leq\left\|y_{k}-P_{\tau} y_{k}\right\|_{\sigma_{0}}+\left\|P_{\tau} y_{k}-v_{k}\right\|_{\sigma_{0}}$.

Because we are assuming that $y_{k}, k=1, \ldots, n$, is of the form $y_{k}(t)=y_{k}^{(1)}(t)+y_{k}^{(2)}(t)$, where $y_{k}^{(1)}(t) \in C^{(m)}([0, T])$ and $y_{k}^{(2)}(t) \in \mathcal{V}_{m}^{\alpha}$, from Lemma 3 we obtain

$\left\|y_{k}-P_{\tau} y_{k}\right\|_{\sigma_{0}} \leq c_{1} \tau_{0}^{m}\left\|\frac{d^{m}}{d t^{m}} y_{k}^{(1)}\right\|_{\sigma_{0}}$.

On the other hand, since $v_{k} \in \mathcal{V}_{m}^{\alpha}$, then $v_{k}=P_{\tau} v_{k}$, and therefore, from Lemma 3, we have

$$
\begin{aligned}
\left\|P_{\tau} y_{k}-v_{k}\right\|_{\sigma_{0}} & \leq \max _{j=1, \ldots, \ell}\left\|\mathcal{L}_{0 j}\right\|_{\sigma_{0}} \sum_{j=1}^{\ell}\left|y_{k}\left(t_{0 j}\right)-v_{k}\left(t_{0 j}\right)\right| \\
& \leq \Delta_{0} \sum_{j=1}^{\ell}\left|y_{k}\left(t_{0 j}\right)-v_{k}\left(t_{0 j}\right)\right|,
\end{aligned}
$$

with $\Delta_{0}$ given by (25). Using (31) and (30) in (29), we obtain

$$
\left\|y_{k}-v_{k}\right\|_{\sigma_{0}} \leq c_{1} \tau_{0}^{m} C_{d}+\Delta_{0} \sum_{j=1}^{\ell}\left|y_{k}\left(t_{0 j}\right)-v_{k}\left(t_{0 j}\right)\right|, \quad k=1, \ldots, n,
$$

where $C_{d}$ is a positive constant defined by $C_{d}=\max _{1 \leq k \leq n}\left\|\frac{d^{m}}{d t^{m}} y_{k}^{(1)}\right\|_{\sigma_{0}}$.

Substituting in (28), we have, for $i=1, \ldots, n$ and $j=1, \ldots, \ell$,

$$
\left|e_{0 j}^{i}\right| \leq \frac{t_{0 j}^{\alpha}}{\Gamma(\alpha+1)}\left(c_{1} C_{d} \tau_{0}^{m} \sum_{k=1}^{n}\left|a_{i k}\right|+\Delta_{0} \sum_{k=1}^{n}\left|a_{i k}\right| \sum_{j=1}^{\ell}\left|e_{0 j}^{k}\right|\right) \text {. }
$$

Then, from the last inequality and from the fact that $\tau_{0} \leq c N^{-1}$, where $c$ is a positive constant that does not depend on $N$ (see [1]), we have

$$
\max _{1 \leq i \leq n}\left|e_{0 j}^{i}\right| \leq C_{1} N^{-m}+C_{2} \sum_{j=1}^{\ell} \max _{1 \leq k \leq n}\left|e_{0 j}^{k}\right|,
$$

where $C_{1}$ and $C_{2}$ are positive constants does not depend on $N$. Hence it follows

$$
\max _{1 \leq j \leq \ell} \max _{1 \leq i \leq n}\left|e_{0 j}^{i}\right| \leq C_{1} N^{-m}+C_{3} \max _{1 \leq j \leq \ell} \max _{1 \leq k \leq n}\left|e_{0 j}^{k}\right| \text {, }
$$

where $C_{3}=\ell C_{2}$.

Hence, for sufficiently large $N$, there exists a positive constant $C_{4}$ such that

$$
\max _{1 \leq j \leq \ell} \max _{1 \leq i \leq n}\left|e_{0 j}^{i}\right| \leq C_{4} N^{-m},
$$

and therefore, from (32) we thus obtain

$\max _{1 \leq k \leq n}\left\|y_{k}-v_{k}\right\|_{\sigma_{0}} \leq C_{5} N^{-m}$,

where $C_{5}$ is a positive constant does not depend on $N$, and the result is proved .

Let us now analyse the error at the remaining subintervals of the mesh.

For each $j=1, \ldots, m$ and $k=1, \ldots, N^{\prime}-1$, we analyse the error at the collocation points $t_{k j}$ :

$\mathbf{e}_{k j}=\left[e_{k j}^{1} e_{k j}^{2} \ldots e_{k j}^{n}\right]^{T}$,

where $e_{k j}^{i}=y_{i}\left(t_{k j}\right)-v_{i}\left(t_{k j}\right), i=1,2, \ldots, n$. 
Lemma 3 Let $\mathbf{y}(t)=\left[y_{k}(t)\right]_{k=1}^{n}$ be the solution of (1)-(2), and $\mathbf{v}(t)=\left[v_{k}(t)\right]_{k=1}^{n}$ the approximate solution obtained by the hybrid collocation method and defined by (24). On each subinterval $\sigma_{k}, k=1, \ldots, N^{\prime}-1$, we have

$$
\max _{1 \leq i \leq n}\left\|y_{i}-v_{i}\right\|_{\sigma_{k}} \leq C N^{-m},
$$

where $C$ is a positive constant that does not depend on $N$.

Proof From (14) and (16) we have, for $j=1, \ldots, m, k=1, \ldots, N^{\prime}-1$ and $i=1, \ldots, n$ :

$$
\begin{aligned}
e_{k j}^{i}= & y_{i}\left(t_{k j}\right)-v_{i}\left(t_{k j}\right)=\frac{1}{\Gamma(\alpha)} \int_{0}^{t_{1}}\left(t_{k j}-s\right)^{\alpha-1} \sum_{l=1}^{n} a_{i l}\left(y_{l}(s)-v_{l}(s)\right) d s \\
& +\frac{1}{\Gamma(\alpha)} \sum_{\gamma=1}^{k-1} \int_{t_{\gamma}}^{t_{\gamma+1}}\left(t_{k j}-s\right)^{\alpha-1} \sum_{l=1}^{n} a_{i l}\left(y_{l}(s)-P_{\gamma}\left(y_{l}\right)(s)+P_{\gamma}\left(y_{l}\right)(s)-v_{l}(s)\right) d s+ \\
& +\frac{1}{\Gamma(\alpha)} \int_{t_{k}}^{t_{k j}}\left(t_{k j}-s\right)^{\alpha-1} \sum_{l=1}^{n} a_{i l}\left(y_{l}(s)-P_{k}\left(y_{l}\right)(s)+P_{k}\left(y_{l}\right)(s)-v_{l}(s)\right) d s,
\end{aligned}
$$

where $P_{\gamma}, \gamma=1, \ldots N^{\prime}-1$, is the interpolation operator defined by (21).

Since $\tau_{i} \leq c N^{-1}$ (see (13)) we have, for $k=1, \ldots, N^{\prime}-1$,

$$
\begin{aligned}
& \int_{t_{\gamma}}^{t_{\gamma+1}}\left(t_{k j}-s\right)^{\alpha-1} d s \leq \bar{c} \tau^{\alpha}(k-\gamma)^{\alpha-1} \leq \bar{c}_{1} N^{-\alpha}(k-\gamma)^{\alpha-1}, \quad \gamma=1, \ldots, k-1, \\
& \int_{t_{k}}^{t_{k j}}\left(t_{k j}-s\right)^{\alpha-1} d s \leq \bar{c} \tau^{\alpha} \leq \bar{c}_{1} N^{-\alpha}, \quad j=1, \ldots, m,
\end{aligned}
$$

where $\bar{c}_{1}$ is a positive constant does not depend on $N$. On the other hand, since $v_{l} \in \mathcal{P}_{m-1}$ then $P_{\gamma}\left(v_{l}\right)(s)=$ $v_{l}(s), s \in \tau_{\sigma}, \quad \sigma=1, \ldots, N^{\prime}-1$, and hence for $s \in \sigma_{\gamma}, \gamma=1,2, \ldots, N^{\prime}-1$ we have

$P_{\sigma}\left(y_{l}\right)(s)-v_{l}(s)=\sum_{j=1}^{m} L_{\gamma j}(s)\left(y_{l}\left(t_{\gamma j}\right)-v_{l}\left(t_{\gamma j}\right)\right), \sigma=1, \ldots, N^{\prime}-1$.

Then, taking modulus and using the above bounds we obtain

$$
\begin{aligned}
\left|e_{k j}^{i}\right| \leq & \frac{1}{\Gamma(\alpha)} \sum_{l=1}^{n}\left|a_{i l}\right|\left\|y_{l}-v_{l}\right\|_{\sigma_{0}} \int_{0}^{t_{1}}\left(t_{k j}-s\right)^{\alpha-1} d s \\
& +\frac{1}{\Gamma(\alpha)} \sum_{l=1}^{n}\left|a_{i l}\right| \sum_{\gamma=1}^{k-1}\left\|y_{l}-P_{\gamma}\left(y_{l}\right)\right\|_{\sigma_{\gamma}} \int_{t_{\gamma}}^{t_{\gamma+1}}\left(t_{k j}-s\right)^{\alpha-1} d s \\
& +\frac{1}{\Gamma(\alpha)} \sum_{l=1}^{n}\left|a_{i l}\right|\left\|y_{l}-P_{k}\left(y_{l}\right)\right\|_{\sigma_{k}} \int_{t_{k}}^{t_{k j}}\left(t_{k j}-s\right)^{\alpha-1} d s \\
& +\frac{m \Delta_{m} \bar{c}_{1}}{\Gamma(\alpha)} N^{-\alpha} \sum_{l=1}^{n}\left|a_{i l}\right| \sum_{\gamma=1}^{k-1}(k-\gamma)^{\alpha-1} \max _{p=1, \ldots, m}\left|e_{\gamma p}^{l}\right| \\
& +\frac{m \Delta_{m} \tau^{\alpha}}{\Gamma(\alpha+1)} \sum_{l=1}^{n}\left|a_{i l}\right| \max _{p=1, \ldots, m}\left|e_{k p}^{l}\right|,
\end{aligned}
$$

where $\Delta_{m}$ is the Lebesgue constant associated with the collocation parameters $c_{1}, \ldots, c_{m}$.

From Theorem 3 follows $\left\|y_{l}-v_{l}\right\|_{\sigma_{0}} \leq C N^{-m}, l=1, \ldots, n$. Let us now investigate what happens with $\left\|y_{l}-P_{\gamma}\left(y_{l}\right)\right\|_{\sigma_{\gamma}}, l=1, \ldots, n, \gamma=1, \ldots, N^{\prime}-1$. 
From the classical interpolation theory and because, as mentioned earlier, $\tau_{i} \leq c N^{-1}, i=1, \ldots, N^{\prime}-1$, we have

$$
\left\|y_{l}-P_{\gamma}\left(y_{l}\right)\right\|_{\sigma_{\gamma}} \leq d_{1} \tau_{\gamma}^{m} \max _{t \in \sigma_{\gamma}}\left|\frac{d^{m} y(t)}{d t^{m}}\right| \leq d_{2} \tau_{\gamma}^{m} \max _{t \in \sigma_{\gamma}}\left|t^{\alpha-m}\right| \leq d_{2} \tau_{\gamma}^{m} t_{\gamma}^{\alpha-m}=d_{2} \tau_{\gamma}^{m}\left(\frac{i_{0}+\gamma-1}{N}\right)^{\frac{m}{\alpha}(\alpha-m)},
$$

where we have also used the argument in [31] that says that if a certain function $f$ for which $f(t)=f_{1}(t)+f_{2}(t)$, where $f_{1} \in C^{m}([0, T])$ and $f_{2} \in V_{m}^{\alpha}$, then $\left|f^{(m)}(t)\right| \leq c t^{\alpha-m}$ for $t \geq t_{1}$.

Using estimate (13) stating that $\tau_{i} \leq c\left(i+i_{0}-2\right)^{\frac{m}{\alpha}-1} N^{-\frac{m}{\alpha}}$, we easily achieve the estimate

$$
\left\|y_{l}-P_{\gamma}\left(y_{l}\right)\right\|_{\sigma_{\gamma}} \leq \bar{c}_{2} N^{-m}, \gamma=1, \ldots, N^{\prime}-1 \text {, }
$$

for some positive constant $\bar{c}_{2}$ that does not depend on $N$.

Using the results (2) and (35) in (34), for $N$ sufficiently large, follows

$$
\max _{i=1, \ldots, n} \max _{p=1, \ldots, m}\left|e_{k p}^{i}\right| \leq \bar{C}_{2} N^{-m}+\bar{C}_{3} N^{-\alpha} \sum_{\gamma=1}^{k-1}(k-\gamma)^{\alpha-1} \max _{i=1, \ldots, n} \max _{p=1, \ldots, m}\left|e_{\gamma p}^{l}\right|
$$

where $\bar{C}_{2}$ and $\bar{C}_{3}$ are positive constants. Applying a standard weakly singular discrete Gronwall inequality, leads to the following result

$$
\max _{i=1, \ldots, n} \max _{p=1, \ldots, m}\left|e_{k p}^{i}\right| \leq \bar{C}_{4} N^{-m} .
$$

On the other hand, using the interpolation error (35) and definition of the interpolation operator $P_{\gamma}$ we obtain, for $\gamma=1, \ldots, N^{\prime}-1$,

$\max _{k=1, \ldots, n}\left\|y_{k}-v_{k}\right\|_{\sigma_{\gamma}} \leq \bar{c}_{2} N^{-m}+m \Delta_{m} \max _{k=1, \ldots, n} \max _{j=1, \ldots, m}\left|e_{\gamma j}^{k}\right|$,

and therefore, from (37) we thus obtain for $k=1, \ldots, n$

$$
\left\|y_{k}-v_{k}\right\|_{\sigma_{\gamma}} \leq \bar{C}_{5} N^{-m}, \gamma=1, \ldots, N^{\prime},
$$

where $\bar{C}_{5}$ is a positive constant does not depend on $N$, and the result is proved.

\section{Numerical Results}

\subsection{Fractional Differential Equations}

In order to illustrate the feasibility of the method, some examples for which the analytical solution is known are presented together with a comparison with the hereafter designated by nonpolynomial method presented in [12]. The numerical error is measured by determining the maximum error at the mesh points $t_{j}$ :

$\varepsilon_{\tau}=\max _{i=1, \ldots, n} \max _{p=1, \ldots, N^{\prime}}\left|y_{i}\left(t_{p}\right)-v_{i}\left(t_{p}\right)\right|$

where $v_{i}$ is the approximate solution, for the $i$-th spatial function, obtained by the hybrid method.

\section{Example 1}

$\left\{\begin{array}{l}D^{\frac{1}{2}} y(t)=\frac{1}{2} y(t), \quad t>0 \\ y(0)=1\end{array}\right.$

whose analytical solution is $y(t)=E_{1 / 2}(0.5 \sqrt{t})$, and 
Example 2

$\left\{\begin{array}{l}D^{\alpha} y_{1}(t)=y_{2}(t) \\ D^{\alpha} y_{2}(t)=-y_{1}(t)-y_{2}(t)+t^{\alpha+1}+\frac{\pi \csc (\pi \alpha) t^{1-\alpha}}{\Gamma(-\alpha-1) \Gamma(2-\alpha)}+\frac{\pi t \csc (\pi \alpha)}{\Gamma(-\alpha-1)},\end{array}\right.$

$y_{1}(0)=0, y_{2}(0)=0$. The analytical solution is given by $y_{1}(t)=t^{1+\alpha}$ and $y_{2}(t)=\pi \alpha(\alpha+1) t \csc (\pi \alpha) / \Gamma(1-\alpha)$.

For the numerical solution of Example 1 we consider the spaces $\mathcal{V}_{2}^{\alpha}, \mathcal{V}_{3}^{\alpha}, \mathcal{V}_{4}^{\alpha}, \mathcal{V}_{5}^{\alpha}, \mathcal{V}_{6}^{\alpha}$.

From Table 1 we observe that the nonpolynomial method provides a better convergence rate for smaller stepsizes, but, the speed-up (SU-ratio between the nonpolynomial and hybrid computational times) obtained with the hybrid method is really high (upt to $450 \times$ ). Note also that the maximum and minimum condition number of the matrices involved $\left(\kappa(A)=\|A\|_{\infty}\left\|A^{-1}\right\|_{\infty}\right)$ obtained for each simulation show that the hybrid method provides better conditioned matrices. Although the $\kappa(A)_{\max }$ looks similar for both methods, it should be remarked that right after the first time-step we obtain the value of $\kappa(A)_{\min }$ for the hybrid method, while for the nonpolynomial method the $\kappa(A)$ is slowly decreasing along time-steps. For $m=4$ it was impossible to obtain convergence for certain values of $N$ in the nonpolynomial method. The number of significant digits lost along the iterative procedure lead to badly conditioned matrices. For the hybrid method that problem could be solved by increasing the number of significant digits (Table 2).

We were able to perform computations up to $m=6$ by using the hybrid method. The simulations were fast and the new method proved to be robust.

Table 1 Hybrid and nonpolynomial collocation methods for Example 1 with three different values of $m$ : values of the maximum of the absolute errors at the mesh points, the experimental orders of convergence $p$ and speed-up (SU)

\begin{tabular}{|c|c|c|c|c|c|c|c|c|c|c|}
\hline \multicolumn{2}{|c|}{ Stepsizes } & \multicolumn{5}{|c|}{ Hybrid $(m=2)$} & \multicolumn{4}{|c|}{ Nonpolynomial $(m=2)$} \\
\hline$N$ & $N^{\prime}$ & $\varepsilon_{\tau}$ & $p$ & SU & $\kappa(A)_{\min }$ & $\kappa(A)_{\max }$ & $\varepsilon_{\tau}$ & $p$ & $\kappa(A)_{\min }$ & $\kappa(A)_{\max }$ \\
\hline 10 & 5 & $1.47 \cdot 10^{-3}$ & - & 10.2 & 1.19 & 16.9 & $5.70 \cdot 10^{-5}$ & - & 4.92 & 26.87 \\
\hline 20 & 11 & $4.45 \cdot 10^{-4}$ & 1.72 & 52.5 & 1.09 & 9.58 & $1.42 \cdot 10^{-5}$ & 2.00 & 3.56 & 16.84 \\
\hline 40 & 25 & $1.21 \cdot 10^{-4}$ & 1.88 & 114.9 & 1.04 & 5.33 & $3.56 \cdot 10^{-6}$ & 2.00 & 2.70 & 10.71 \\
\hline 80 & 54 & $3.15 \cdot 10^{-5}$ & 1.94 & 175.7 & 1.02 & 3.69 & $8.90 \cdot 10^{-7}$ & 2.00 & 2.14 & 7.01 \\
\hline 160 & 116 & $8.05 \cdot 10^{-6}$ & 1.97 & 156.5 & 1.01 & 2.67 & $2.23 \cdot 10^{-7}$ & 2.00 & 1.78 & 4.78 \\
\hline \multicolumn{2}{|c|}{ Stepsizes } & \multicolumn{5}{|c|}{ Hybrid $(m=3)$} & \multicolumn{4}{|c|}{ Nonpolynomial $(m=3)$} \\
\hline$N$ & $\mathrm{~N}^{\prime}$ & $\varepsilon_{\tau}$ & $p$ & SU & $\kappa(A)_{\min }$ & $\kappa(A)_{\max }$ & $\varepsilon_{\tau}$ & $p$ & $\kappa(A)_{\min }$ & $\kappa(A)_{\max }$ \\
\hline 10 & 4 & $5.01 \cdot 10^{-4}$ & - & 39.9 & 1.47 & 135.5 & $1.50 \cdot 10^{-9}$ & - & 3.36 & 115.52 \\
\hline 20 & 8 & $7.65 \cdot 10^{-5}$ & 2.71 & 270.3 & 1.23 & 88.04 & $1.50 \cdot 10^{-10}$ & 3.33 & 2.44 & 59.97 \\
\hline 40 & 19 & $1.05 \cdot 10^{-5}$ & 2.86 & 363.0 & 1.10 & 35.42 & $1.60 \cdot 10^{-11}$ & 3.23 & 1.93 & 32.45 \\
\hline 80 & 42 & $1.39 \cdot 10^{-6}$ & 2.92 & 450.7 & 1.05 & 19.43 & $1.80 \cdot 10^{-12}$ & 3.16 & 1.62 & 18.37 \\
\hline 160 & 92 & $1.78 \cdot 10^{-7}$ & 2.96 & 406.4 & 1.02 & 11.15 & $2.07 \cdot 10^{-13}$ & 3.12 & 1.42 & 10.93 \\
\hline \multicolumn{2}{|c|}{ Stepsizes } & \multicolumn{5}{|c|}{ Hybrid $(m=4)$} & \multicolumn{4}{|c|}{ Nonpolynomial $(m=4)$} \\
\hline$N$ & $N^{\prime}$ & $\varepsilon_{\tau}$ & $p$ & SU & $\kappa(A)_{\min }$ & $\kappa(A)_{\max }$ & $\varepsilon_{\tau}$ & $p$ & $\kappa(A)_{\min }$ & $\kappa(A)_{\max }$ \\
\hline 10 & 3 & $2.23 \cdot 10^{-4}$ & - & 67.0 & 2.14 & 426.1 & $3.83 \cdot 10^{-13}$ & - & 1.48 & 253.57 \\
\hline 20 & 7 & $1.77 \cdot 10^{-5}$ & 3.65 & 122.2 & 1.38 & 148.5 & $1.64 \cdot 10^{-14}$ & 4.54 & 1.31 & 129.68 \\
\hline 40 & 15 & $1.23 \cdot 10^{-6}$ & 3.84 & - & 1.18 & 85.18 & - & - & - & - \\
\hline 80 & 34 & $8.14 \cdot 10^{-8}$ & 3.92 & - & 1.08 & 41.44 & - & - & - & - \\
\hline 160 & 76 & $5.23 \cdot 10^{-9}$ & 3.96 & - & 1.04 & 21.26 & - & - & - & - \\
\hline
\end{tabular}


Table 2 Hybrid collocation method for Example 1 with two different values of $m$ : values of the maximum of the absolute errors at the mesh points and the experimental orders of convergence $p$

\begin{tabular}{|c|c|c|c|c|c|}
\hline \multicolumn{2}{|c|}{ Stepsizes } & \multicolumn{4}{|c|}{ Hybrid $(m=5)$} \\
\hline$N$ & $N^{\prime}$ & $\varepsilon_{\tau}$ & $p$ & $\kappa(A)_{\min }$ & $\kappa(A)_{\max }$ \\
\hline 10 & 3 & $2.13 \cdot 10^{-5}$ & - & 3.35 & $1.05 \cdot 10^{6}$ \\
\hline 20 & 6 & $8.51 \cdot 10^{-7}$ & 4.65 & 1.85 & $5.36 \cdot 10^{5}$ \\
\hline 40 & 13 & $2.96 \cdot 10^{-8}$ & 4.85 & 1.37 & $2.63 \cdot 10^{5}$ \\
\hline 80 & 29 & $9.78 \cdot 10^{-10}$ & 4.92 & 1.17 & $1.23 \cdot 10^{5}$ \\
\hline 160 & 64 & $3.16 \cdot 10^{-11}$ & 4.95 & 1.08 & $6.10 \cdot 10^{4}$ \\
\hline \multicolumn{2}{|c|}{ Stepsizes } & \multicolumn{4}{|c|}{ Hybrid $(m=6)$} \\
\hline$N$ & $N^{\prime}$ & $\varepsilon_{\tau}$ & $p$ & $\kappa(A)_{\min }$ & $\kappa(A)_{\max }$ \\
\hline 10 & 2 & $1.38 \cdot 10^{-5}$ & - & 128.46 & $4.81 \cdot 10^{8}$ \\
\hline 20 & 5 & $3.72 \cdot 10^{-7}$ & 5.21 & 14.95 & $1.07 \cdot 10^{8}$ \\
\hline 40 & 11 & $6.66 \cdot 10^{-9}$ & 5.80 & 2.48 & $4.79 \cdot 10^{7}$ \\
\hline 80 & 25 & $1.16 \cdot 10^{-10}$ & 5.84 & 1.63 & $2.05 \cdot 10^{7}$ \\
\hline
\end{tabular}

Table 3 Error, speed-up (SU) and convergence order $(p)$ obtained for the numerical solution of Example 2 using the Hybrid and nonpolynomial collocation methods, for $\alpha=1 / 4,1 / 2,2 / 3$

\begin{tabular}{|c|c|c|c|c|c|c|c|c|c|c|c|}
\hline \multirow{2}{*}{$\begin{array}{l}\text { Stepsizes } \\
N\end{array}$} & \multicolumn{3}{|c|}{ Hybrid $(\alpha=1 / 4)$} & \multicolumn{4}{|c|}{ Hybrid $(\alpha=1 / 2)$} & \multicolumn{4}{|c|}{ Hybrid $(\alpha=2 / 3)$} \\
\hline & $N^{\prime}$ & $\varepsilon_{\tau}$ & $p$ & $N^{\prime}$ & $\varepsilon_{\tau}$ & $p$ & SU & $N^{\prime}$ & $\varepsilon_{\tau}$ & $p$ & SU \\
\hline 64 & 15 & $2.11 \cdot 10^{-6}$ & 3.70 & 26 & $1.94 \cdot 10^{-7}$ & 3.86 & 56.6 & 32 & $5.53 \cdot 10^{-8}$ & 3.90 & 142.2 \\
\hline 128 & 34 & $1.45 \cdot 10^{-7}$ & 3.86 & 59 & $1.27 \cdot 10^{-8}$ & 3.93 & 51.0 & 71 & $3.58 \cdot 10^{-9}$ & 3.95 & 162.4 \\
\hline 256 & 75 & $9.43 \cdot 10^{-9}$ & 3.94 & 128 & $8.13 \cdot 10^{-10}$ & 3.97 & 52.2 & 155 & $2.28 \cdot 10^{-10}$ & 3.97 & 170.2 \\
\hline 512 & 166 & $6.00 \cdot 10^{-10}$ & 3.97 & 278 & $5.14 \cdot 10^{-11}$ & 3.98 & 60.0 & 331 & $1.44 \cdot 10^{-11}$ & 3.99 & 173.4 \\
\hline
\end{tabular}

Next we consider the second example where a systems of equations is considered.

The numerical method was used to solve Example 2 with $m=4$ by considering $\alpha=1 / 4,1 / 2,2 / 3$. The error and the experimental convergence order are listed in Table 3 . As expected we have obtained an optimal convergence order that is independent of $\alpha$.

We obtained speed-ups that go up to $173 \times$. Note that SU is not provided for the case $\alpha=1 / 4$ because the simulation time for the nonpolynomial method became really high.

Now that we have shown the feasibility of using the hybrid method for the solution of systems of ordinary fractional differential equations, we will analise its application to time-fractional diffusion equations.

\subsection{Case Study: The Time-Fractional Diffusion Equation}

We will now apply the hybrid method in the numerical solution of the time fractional diffusion equation given by Eq. (3) together with the initial and boundary conditions given by Eqs. (4) and (5). In order to do that, we use the method of lines to convert (3)-(5) into a system of fractional ordinary differential equations. 
We consider a uniform space mesh on the interval $[0, L]$, defined by the gridpoints $x_{i}=i h, i=0, \ldots, n$, where $h=\frac{L}{n}$, and we approximate the space derivative by the second order finite difference:

$\frac{\partial^{2} u\left(x_{i}, t\right)}{\partial x^{2}}=\frac{u\left(x_{i+1}, t\right)-2 u\left(x_{i}, t\right)+u\left(x_{i-1}, t\right)}{h^{2}}+\mathcal{O}\left(h^{2}\right), \quad i=1, \ldots, n-1$.

Neglecting the $\mathcal{O}\left(h^{2}\right)$ terms, we then obtain the following system of $n-1$ fractional differential equations:

$\frac{\partial^{\alpha} y_{i}(t)}{\partial t^{\alpha}}=D_{\alpha} \frac{y_{i+1}(t)-2 y_{i}(t)+y_{i-1}(t)}{h^{2}}+f\left(x_{i}, t\right), \quad i=1, \ldots, n-1$,

where $y_{i}(t) \approx u\left(x_{i}, t\right)$.

Note that from the boundary conditions (5), we have $y_{0}(t)=u_{0}, y_{n}(t)=u_{L}$ and from the initial condition (4), we obtain:

$y_{i}(0)=g\left(x_{i}\right), \quad i=1, \ldots, n-1$,

and therefore, the solution of the $n-1$ initial value problems (43)-(44) may be determined by using any initial value problem solver. The problem to solve may be outlined as follows.

For each $n \in \mathbf{N}$ and $t \geq 0$ we define

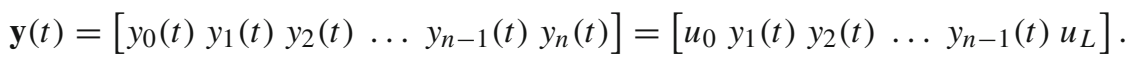

Thus, the system (43), (44) can be rewritten as follows

$\left\{\begin{array}{l}\frac{\partial^{\alpha} y_{i}(t)}{\partial t^{\alpha}}=F_{i}(t, \mathbf{y}(t)), \quad i=1, \ldots, n-1, \\ y_{i}(0)=g\left(x_{i}\right), \quad i=1, \ldots, n-1,\end{array}\right.$

where each function $F_{i}$ is defined by

$F_{i}(t, \mathbf{y}(t))=D_{\alpha} \frac{y_{i+1}(t)-2 y_{i}(t)+y_{i-1}(t)}{h^{2}}+f\left(x_{i}, t\right), i=1, \ldots, n-1, t>0$.

Hence, we end up with a system of $(n-1)$ fractional ordinary differential equations which is solved by using the hybrid collocation method presented before.

In order to illustrate the feasibility and performance of the method we will now compare the results obtained with this method with the ones obtained with the method in [14]. The numerical error is measured by determining the maximum error at the mesh points $\left(x_{i}, t_{j}\right)$ :

$\varepsilon_{h, \tau}=\max _{i=1, \ldots, n, j=1, \ldots, N^{\prime}}\left|u\left(x_{i}, t_{j}\right)-y_{i}\left(t_{j}\right)\right|, n=\frac{L}{h}$

where $y_{i}$ is the numerical solution obtained for the $i$-th spatial function and $u\left(x_{i}, t_{j}\right)$ is the exact solution evaluated at points $\left(x_{i}, t_{j}\right)$. We consider the following examples:

Example 3

$\left\{\begin{array}{l}\frac{\partial^{\alpha} u(x, t)}{\partial t^{\alpha}}=\frac{\partial^{2} u(x, t)}{\partial x^{2}}+\frac{\Gamma(4+\alpha)}{6} x^{4}(2-x) t^{3}-4 x^{2}(6-5 x) t^{3+\alpha}, \quad t>0,0 \leq x \leq 2, \\ u(x, 0)=0, \\ u(0, t)=u(2, t)=0,\end{array}\right.$

whose analytical solution is $u(x, t)=x^{4}(2-x) t^{3+\alpha}$.

\section{Example 4}

$\left\{\begin{array}{l}\frac{\partial^{\alpha} u(x, t)}{\partial t^{\alpha}}=\frac{\partial^{2} u(x, t)}{\partial x^{2}}+4 t^{\alpha} x^{2}(5 x-3)+\frac{\pi(x-1) x^{4} \csc (\pi \alpha)}{\Gamma(-\alpha)}, \quad t>0,0 \leq x \leq 1, \\ u(x, 0)=0, \\ u(0, t)=u(1, t)=0,\end{array}\right.$

whose analytical solution is $u(x, t)=x^{4}(1-x) t^{\alpha}$. 
Table 4 Hybrid and nonpolynomial collocation methods on the space $V_{2}^{\alpha}$ for Example 3: values of the maximum of the absolute errors at the mesh points and experimental orders of convergence

\begin{tabular}{|c|c|c|c|c|c|c|c|c|}
\hline \multicolumn{3}{|c|}{ Stepsizes } & \multicolumn{6}{|c|}{ Hybrid $(\alpha=2 / 3)$} \\
\hline$N$ & $N^{\prime}$ & $h$ & $\varepsilon_{h, \tau}$ & $p=q$ & $\mathrm{SU}$ & $\kappa(A)_{\min }$ & $\kappa(A)_{\max }$ & $\operatorname{dim}(A)$ \\
\hline 8 & 4 & 0.125 & $3.17 \cdot 10^{-1}$ & - & 45.4 & 86.7 & $1.14 \cdot 10^{+4}$ & $75 / 30$ \\
\hline 16 & 10 & 0.0625 & $9.59 \cdot 10^{-2}$ & 1.73 & 42.8 & 151.7 & $2.60 \cdot 10^{+4}$ & $155 / 62$ \\
\hline 32 & 22 & 0.0313 & $2.53 \cdot 10^{-2}$ & 1.92 & 247.4 & 281.1 & $6.57 \cdot 10^{+4}$ & $315 / 126$ \\
\hline 64 & 48 & 0.0156 & $6.21 \cdot 10^{-3}$ & 2.02 & 142.1 & 494.8 & $1.73 \cdot 10^{+5}$ & $635 / 254$ \\
\hline \multicolumn{9}{|c|}{ Nonpolynomial $(\alpha=2 / 3)$} \\
\hline $\bar{N}$ & $\varepsilon_{h, \tau}$ & $p=q$ & \multicolumn{6}{|l|}{$\kappa(A)_{\min }$} \\
\hline 8 & $2.61 \cdot 10^{-2}$ & 1.97 & \multicolumn{6}{|l|}{$2.93 \cdot 10^{+2}$} \\
\hline 16 & $6.54 \cdot 10^{-3}$ & 2.00 & \multicolumn{6}{|l|}{$8.19 \cdot 10^{+2}$} \\
\hline 32 & $1.64 \cdot 10^{-3}$ & 2.00 & \multicolumn{6}{|l|}{$2.20 \cdot 10^{+3}$} \\
\hline 64 & $4.09 \cdot 10^{-4}$ & 2.00 & \multicolumn{6}{|l|}{$6.64 \cdot 10^{+3}$} \\
\hline \multicolumn{3}{|c|}{ Stepsizes } & \multicolumn{6}{|c|}{ Hybrid $(\alpha=1 / 2)$} \\
\hline$N$ & $N^{\prime}$ & $h$ & $\varepsilon_{h, \tau}$ & $p=q$ & SU & $\kappa(A)_{\min }$ & $\kappa(A)_{\max }$ & $\operatorname{dim}(A)$ \\
\hline 8 & 4 & 0.125 & $4.16 \cdot 10^{-1}$ & - & 30.1 & 101.2 & 744.3 & $60 / 30$ \\
\hline 16 & 8 & 0.0625 & $1.39 \cdot 10^{-1}$ & 1.58 & 32.2 & 251.1 & $2.36 \cdot 10^{+2}$ & $124 / 62$ \\
\hline 32 & 19 & 0.0313 & $3.95 \cdot 10^{-2}$ & 1.81 & 54.5 & 529.1 & $5.88 \cdot 10^{+3}$ & $252 / 126$ \\
\hline 64 & 42 & 0.0156 & $1.03 \cdot 10^{-2}$ & 1.94 & 167.8 & 1144 & $1.67 \cdot 10^{+4}$ & $508 / 254$ \\
\hline \multicolumn{9}{|c|}{ Nonpolynomial $(\alpha=1 / 2)$} \\
\hline $\bar{N}$ & $\varepsilon_{h, \tau}$ & $p=q$ & \multicolumn{6}{|l|}{$\kappa(A)_{\min }$} \\
\hline 8 & $2.92 \cdot 10^{-2}$ & 1.99 & \multicolumn{6}{|l|}{$2.27 \cdot 10^{+2}$} \\
\hline 16 & $7.31 \cdot 10^{-3}$ & 2.00 & \multicolumn{6}{|l|}{$6.84 \cdot 10^{+3}$} \\
\hline 32 & $1.83 \cdot 10^{-3}$ & 2.00 & \multicolumn{6}{|l|}{$2.04 \cdot 10^{+3}$} \\
\hline 64 & $4.57 \cdot 10^{-4}$ & 2.00 & \multicolumn{6}{|l|}{$6.08 \cdot 10^{+3}$} \\
\hline \multicolumn{3}{|c|}{ Stepsizes } & \multicolumn{6}{|c|}{ Hybrid $(\alpha=1 / 3)$} \\
\hline$N$ & $N^{\prime}$ & $h$ & $\varepsilon_{h, \tau}$ & $p=q$ & $\mathrm{SU}$ & $\kappa(A)_{\min }$ & $\kappa(A)_{\max }$ & $\operatorname{dim}(A)$ \\
\hline 8 & 3 & 0.125 & $5.75 \cdot 10^{-1}$ & - & 116 & 125.1 & $3.26 \cdot 10^{+6}$ & $90 / 30$ \\
\hline 16 & 6 & 0.0625 & $2.21 \cdot 10^{-1}$ & 1.38 & 173 & 372.6 & $1.10 \cdot 10^{+7}$ & $186 / 62$ \\
\hline 32 & 15 & 0.0313 & $6.90 \cdot 10^{-2}$ & 1.68 & 629.4 & 934.0 & $2.72 \cdot 10^{+7}$ & $378 / 126$ \\
\hline 64 & 32 & 0.0156 & $1.92 \cdot 10^{-2}$ & 1.85 & 198.2 & 2699 & $8.88 \cdot 10^{+7}$ & $762 / 254$ \\
\hline 128 & 71 & 0.0078 & $5.00 \cdot 10^{-3}$ & 1.94 & - & 7278 & $2.86 \cdot 10^{+8}$ & $1530 / 510$ \\
\hline \multicolumn{9}{|c|}{ Nonpolynomial $(\alpha=1 / 3)$} \\
\hline $\bar{N}$ & $\varepsilon_{h, \tau}$ & $p=q$ & $\kappa(A)_{\min }$ & & & & & \\
\hline 8 & $3.18 \cdot 10^{-2}$ & 1.99 & $5.81 \cdot 10^{+3}$ & & & & & \\
\hline 16 & $8.00 \cdot 10^{-3}$ & 1.99 & $1.66 \cdot 10^{+4}$ & & & & & \\
\hline 32 & $2.00 \cdot 10^{-3}$ & 2.00 & $5.07 \cdot 10^{+4}$ & & & & & \\
\hline 64 & $5.00 \cdot 10^{-4}$ & 2.00 & $1.67 \cdot 10^{+5}$ & & & & & \\
\hline
\end{tabular}


The numerical results obtained for Example 3 on the spaces $V_{2, \tau}^{\alpha}$ and $V_{3, \tau}^{\alpha}$ are now presented.

For each case, the estimates for the time and space rates of convergence were computed and denoted by $p$ and $q$, respectively.

In Table 4 we show the numerical results obtained by the described hybrid method and the nonpolynomial method on the space $V_{2}^{\alpha}$ considering three different values of $\alpha$. As expected we have determined experimentally $p \sim 2$ (not dependent on the order of the fractional derivative) and $q \sim 2$.

We also present the speed-up, and the maximum and minimum condition number $\left(\kappa(A)=\|A\|_{\infty}\left\|A^{-1}\right\|_{\infty}\right)$ obtained for each simulation. We observe that the hybrid method allows one to obtain the same order of convergence with a less computational effort, with the speed-up increasing with the mesh refinement. Note that in some cases we managed to obtain speed-ups of $173 x$. We may also conclude that SU increases with the dimension of the space $V_{m}^{\alpha}$. Regarding the condition number, we have that $\kappa(A)_{\max }$ is the same for both the methods, and, this value is obtained only for the first iteration in time. For the hybrid method the value of $\kappa(A)$ for the remaining iterations remains constant, and is given by $\kappa(A)_{\min }$. For the nonpolynomial method the $\kappa(A)$ decreases along iterations, being its minimum value achieved only in the last iteration. Note the difference of the value of $\kappa(A)_{\min }$ in the two methods, being the condition number really low for the hybrid method. This makes this method more robust, since less significant digits will be lost along the numerical procedure. We also present in this table the dimension of the matrices obtained for the first and remaining time intervals $(\operatorname{dim}(A))$. This is denoted by $a / b$ with $a \times a$ the dimension of the matrix for the first time interval and $b \times b$ the dimension of the matrix for the remaining intervals (for the hybrid method). It should be remarked that $\operatorname{dim}(A)=a \times a$ for the nonpolynomial method (in all time intervals), making this method significantly slower when compared to hybrid method.

We have tested higher orders of convergence by considering the space $V_{3}^{1 / 2}$ for Example 3. The results are shown in Table 5.

It is expected that as we refine further the mesh in time, the influence of the singularity at $t=0$ may be felt in other intervals adjacent to the first interval, where the polynomials may not capture well the behavior of the solution. Therefore, we have considered a new example (Example 4) where the singularity is stronger than in the previous case (Example 3).

Table 5 Hybrid and nonpolynomial collocation methods on the space $V_{3}^{1 / 2}$ for Example 3: values of the maximum of the absolute errors at the mesh points and the experimental order of convergence $p$ related with the stepsizes $\tau=(h)^{2 / 3}(\mathrm{~m}=3)$, and $h$

\begin{tabular}{lllll}
\hline Stepsizes & & & \multicolumn{2}{c}{ Hybrid $(\alpha=1 / 2)-V_{3}^{1 / 2}$} \\
\cline { 1 - 2 }$N$ & $N^{\prime}$ & $h$ & $\varepsilon_{h, \tau}$ & $p$ \\
\hline 16 & 6 & 0.0156 & $2.13 \cdot 10^{-2}$ & - \\
25 & 11 & 0.0078 & $6.58 \cdot 10^{-3}$ & 2.55 \\
40 & 19 & 0.0039 & $1.78 \cdot 10^{-3}$ & 2.83 \\
64 & 32 & 0.00195 & $4.60 \cdot 10^{-4}$ & 2.93
\end{tabular}

Table 6 Hybrid collocation method on the space $V_{1}^{1 / 3}$ for Example 4: values of the maximum of the absolute errors at the mesh points and the experimental orders of convergence $p$ and $q$ related with the stepsizes $\tau=(h)^{2}$ and $h$

\begin{tabular}{llllll}
\hline Stepsizes & & & \multicolumn{2}{l}{ Space $V_{1}^{1 / 3}$} & $p$ \\
\hline$N$ & $N^{\prime}$ & $h$ & $\varepsilon_{h, \tau}$ & - \\
\hline 16 & 10 & 0.1 & $2.25 \cdot 10^{2}$ & - \\
64 & 48 & 0.0208 & $6.15 \cdot 10^{-3}$ & 0.93 & 1.87 \\
256 & 216 & 0.0046 & $1.55 \cdot 10^{-3}$ & 1.00 & 2.00 \\
512 & 448 & 0.0022 & $4.20 \cdot 10^{-4}$ & 2.00 \\
\hline
\end{tabular}


Table 7 Hybrid collocation method on the spaces $V_{2}^{1 / 3}, V_{2}^{1 / 4}$ and $V_{2}^{1 / 6}$ for Example 4: values of the maximum of the absolute errors at the mesh points and the experimental orders of convergence $p$ and $q$ related with the stepsizes $\tau$ and $h$

\begin{tabular}{|c|c|c|c|c|c|c|c|c|c|c|}
\hline \multicolumn{5}{|c|}{ Stepsizes } & \multicolumn{2}{|l|}{ Space $V_{2}^{1 / 3}$} & \multicolumn{2}{|l|}{$\underline{\text { Space } V_{2}^{1 / 4}}$} & \multicolumn{2}{|l|}{ Space $V_{2}^{1 / 6}$} \\
\hline$N$ & $N_{1 / 3}^{\prime}$ & $N_{1 / 4}^{\prime}$ & $N_{1 / 6}^{\prime}$ & $h$ & $\varepsilon_{h, \tau}$ & $p=q$ & $\varepsilon_{h, \tau}$ & $p=q$ & $\varepsilon_{h, \tau}$ & $p=q$ \\
\hline 8 & 3 & 2 & 2 & 0.125 & $5.46 \cdot 10^{-3}$ & - & $5.49 \cdot 10^{-3}$ & - & $4.93 \cdot 10^{-3}$ & - \\
\hline 16 & 6 & 5 & 4 & 0.063 & $1.40 \cdot 10^{-3}$ & 2.02 & $1.40 \cdot 10^{-3}$ & 2.03 & $1.17 \cdot 10^{-3}$ & 2.07 \\
\hline 32 & 15 & 12 & 9 & 0.031 & $3.55 \cdot 10^{-4}$ & 2.01 & $3.55 \cdot 10^{-4}$ & 2.02 & $2.85 \cdot 10^{-4}$ & 2.04 \\
\hline 64 & 32 & 26 & 19 & 0.016 & $8.93 \cdot 10^{-5}$ & 2.00 & $9.00 \cdot 10^{-5}$ & 2.00 & $7.04 \cdot 10^{-5}$ & 2.02 \\
\hline
\end{tabular}

We have considered the spaces $V_{1}^{\alpha}$ and $V_{2}^{\alpha}$ with $\alpha=1 / 3,1 / 4,1 / 6$.

Table 6 shows the absolute errors at the mesh points and convergence rates for both time and space. It can be seen that as we increase the time mesh refinement the method can still deal with the singularity, presenting us with the expected convergence rates. In order to further validate the numerical method we have also considered the spaces $V_{2}^{1 / 3}, V_{2}^{1 / 4}$ and $V_{2}^{1 / 6}$. These results are shown in Table 7. Again, the hybrid method could accurately deal with the low values of $\alpha$, providing the expected convergence rates. We observe that the singularity could not affect other intervals besides the first, at least for the examples considered in this work.

\section{Conclusions}

In this work we have derived a hybrid collocation method that can deal with both smooth and nonsmooth solutions of systems of fractional differential equations. In this hybrid collocation method we combine a nonpolynomial collocation method used near the singular point, the origin, and a graded piecewise polynomial collocation method used for the rest of the domain. We prove the optimal order of global convergence, not depending on $\alpha$, for this method applied to a system of fractional differential equations of order $\alpha, 0<\alpha<1$. Several numerical examples are presented to demonstrate the effectiveness of the proposed method and to compare it with the nonpolynomial collocation method. We conclude that the proposed method is much less time consuming than the nonpolynomial collocation method in $[12,14]$. The numerical technique can also be used to solve the time fractional diffusion equation, using the method of lines. For this type of fractional partial differential equation the numerical results suggest that we obtain an optimal order of convergence in time corresponding to the order of the time integrator solver and second order convergence in space, which corresponds to the order of the discretization of the space derivative.

Acknowledgements L.L. Ferrás would like to thank FCT - Fundação para a Ciência e a Tecnologia, I.P. (Portuguese Foundation for Science and Technology) for financial support through the scholarship SFRH/BPD/100353/2014 and Project UID-MAT-00013/2013. M.L. Morgado aknowledges the financial support of FCT, through the Project UID/Multi/04621/2019 of CEMAT/IST-ID, Center for Computational and Stochastic Mathematics, Instituto Superior Técnico, University of Lisbon. This work was also partially supported by FCT through the Project UID/MAT/00297/2019 (Centro de Matemática e Aplicações).

\section{References}

1. Cao, Y., Herdman, T., Xu, Y.: A hybrid collocation method for Volterra integral equations with weakly singular kernels. SIAM J. Numer. Anal. 41, 364-381 (2003)

2. Chen, C.M., Liu, F., Turner, I., Anh, V.: A Fourier method for the fractional diffusion equation describing sub-diffusion. J. Comput. Phys. 227, 886-897 (2007)

3. Chen, C.M., Liu, F., Burrage, K.: Finite difference methods and a Fourier analysis for the fractional reaction-subdiffusion equation. Appl. Math. Comput. 198, 754-769 (2008)

4. Cui, M.: Compact finite difference method for the fractional diffusion equation. J. Comput. Phys. 228, $7792-7804$ (2009) 
5. Diethelm, K., Ford, N.J.: Analysis of fractional differential equations. J. Math. Anal. Appl. 265, 229-248 (2002)

6. Diethelm, K.: The Analysis of Fractional Differential Equations: An Application-Oriented Exposition Using Differential Operators of Caputo Type. Springer, New York (2010)

7. Diethelm, K.: An algorithm for the numerical solution of differential equations of fractional order. Electr. Trans. Numer. Anal. 5, 1-6 (1997)

8. Diethelm, K., Ford, J.M., Ford, N.J., Weilbeer, M.: Pitfalls in fast numerical solvers for fractional differential equations. J. Comput. Appl. Math. 186, 482-503 (2006)

9. Diethelm, K., Ford, N.J.: Volterra integral equations and fractional calculus: do neighboring solutions intersect? J. Integral Equ. Appl. 1, 25-37 (2012)

10. Ford, N.J., Connolly, J.A.: Comparison of numerical methods for fractional differential equations. Commun. Pure Appl. Anal. 5, 289 (2006)

11. Ford, N., Morgado, M.: Fractional boundary value problems: analysis and numerical methods. Fract. Calc. Appl. Anal. 14, 554-567 (2011)

12. Ford, N.J., Morgado, M.L., Rebelo, M.: Nonpolynomial collocation approximation of solutions to fractional differential equations. Fract. Calc. Appl. Anal. 16, 874-891 (2013)

13. Ford, N.J., Yan, Y.: An approach to construct higher order time discretization schemes for time fractional partial differential equations with nonsmooth data. Fract. Calc. Appl. Anal. 20, 1076-1105 (2017)

14. Ferrás, L.L., Ford, N.J., Morgado, M.L., Rebelo, M.: A numerical method for the solution of the time-fractional diffusion equation. In: Murgante, B. et al. (eds.) Computational Science and Its Applications - ICCSA 2014. ICCSA 2014. Lecture Notes in Computer Science, vol. 8579. Springer, Cham (2014)

15. Gao, G., Sun, Z.Z.: A compact finite difference scheme for the fractional sub-diffusion equations. J. Comput. Phys. 230, 586-595 (2011)

16. Gorenflo, R., Mainardi, F., Moretti, D., Paradisi, P.: Time fractional diffusion: a discrete random walk approach. Nonlinear Dyn. 29, 129-143 (2002)

17. Gu, Y.T., Zhuang, P.: Anomalous sub-diffusion equations by the meshless collocation method. Aust. J. Mech. Eng. 10, 1-8 (2012)

18. Fenghui, H.: A time-space collocation spectral approximation for a class of time fractional differential equations. Int. J. Differ. Equ. 2012, 1-19 (2012). Article ID 495202

19. Langlands, T.A.M., Henry, B.I.: The accuracy and stability of an implicit solution method for the fractional diffusion equation. J. Comput. Phys. 205(2), 719-736 (2005)

20. Lin, Y., Chuanju, X.: Finite difference/spectral approximations for the time-fractional diffusion equation. J. Comput. Phys. 225, 1533-1552 (2007)

21. Liu, F., Yang, C., Burrage, K.: Numerical method and analytical technique of the modified anomalous subdiffusion equation with a nonlinear source term. J. Comput. Appl. Math. 231, 160-176 (2009)

22. Luchko, Y.: Some uniqueness and existence results for the initial-boundary-value problems for the generalized time-fractional diffusion equation. Comput. Math. Appl. 59, 1766-1772 (2010)

23. Luchko, Y.: Maximum principle and its application for the time-fractional diffusion equations. Fract. Calc. Appl. Anal. 14, 110-124 (2011)

24. Luchko, Y.: Initial-boundary-value problems for the one-dimensional time-fractional diffusion equation. Fract. Calc. Appl. Anal. 15, 141-160 (2012)

25. Murio, D.A.: Implicit finite difference approximation for time fractional diffusion equations. Comput. Math. Appl. 5, 1138-1145 (2008)

26. Mainardi, F.: Fractional diffusive waves in viscoelastic solids. In: Wegner, J. I., Norwood, F. R. (eds.) Nonlinear Waves in Solids, ASME Book No. AMR 137 93-97, Fairfield (1995)

27. Mainardi, F.: Some basic problems in continuum and statistical mechanics. In: Carpinteri, A., Mainardi, F. (eds.) Fractals and Fractional Calculus in Continuum Mechanics. Springer, Wien (1997)

28. Mainardi, F., Pagnini, G., Gorenflo, R.: Some aspects of fractional diffusion equations of single and distributed order. Appl. Math. Comput. 187, 295-305 (2007)

29. Mainardi, F.: The time fractional diffusion-wave equation. Radiophys. Quant. Electron. 38, 13-24 (1995)

30. Nigmatullin, R.: The realization of the generalized transfer equation in a medium with fractal geometry. Phys. Status Solidi 133, 425-430 (1986)

31. Rice, J.: On the degree of convergence of nonlinear spline approximation. In: Schoenberg, I.J. (ed.) Approximations with Special Emphasis on Spline Functions, pp. 349-369. Academic Press, New York (1969)

32. Richardson, L.F.: Atmospheric diffusion shown on a distance-neighbour graph. Proc. R. Soc. Lond. Ser. A Contain. Pap. Math. Phys. Character JSTOR 110, 709-737 (1926)

33. Schneider, W., Wyss, W.: Fractional diffusion and wave equations. J. Math. Phys. 30, 134-144 (1989)

34. Stynes, M., O'Riordan, E., Gracia, L.J.: Error analysis of a finite difference method on graded meshes for a time-fractional diffusion equation. SIAM J. Numer. Anal. 55, 1057-1079 (2017)

35. Sun, H.G., Chen, W., Sze, K.Y.: A semi-discrete finite element method for a class of time-fractional diffusion equations. Philos. Trans. R. Soc. A 371, 20120268 (2013)

36. Wyss, W.: The fractional diffusion equation. J. Math. Phys. 27, 27-82 (1986) 
37. YingJun, J., JingTangm, M.A.: Moving finite element methods for time fractional partial differential equations. Sci. China Math. 56, 1287-1300 (2013)

38. Yuste, S.B., Acedo, L.: An explicit finite difference method and a new von Neumann type stability analysis for fractional diffusion equations. SIAM J. Numer. Anal. 42, 1862-1874 (2005)

39. Yuste, S.B.: Weighted average finite difference methods for fractional diffusion equations. J. Comput. Phys. 216, 264-274 (2006)

40. Zhao, X., Sun, Z.Z.: A box-type scheme for fractional sub-diffusion equation with Neumann boundary conditions. J. Comput. Phys. 230, 6061-6074 (2011)

Publisher's Note Springer Nature remains neutral with regard to jurisdictional claims in published maps and institutional affiliations. 